\title{
Causal Association between Bone Mineral Density and Osteoarthritis: A Mendelian Randomization Study
}

\author{
Gwan Gyu Song, Young Ho Lee \\ Department of Rheumatology, Korea University College of Medicine, Seoul, Korea
}

\begin{abstract}
Objective. To examine whether bone mineral density (BMD) is causally associated with osteoarthritis (OA). Methods. We performed a two-sample Mendelian randomization (MR) analysis using the inverse-variance weighting (IVW), weighted median, and MR-Egger regression methods. We used publicly available summary statistics datasets of a genome-wide association study (GWAS) on femur neck (FN) BMD of individuals of European ancestry as the exposure and a GWAS for non-cancer illness code self-reported: OA from the individuals included in the UK Biobank as the outcome. Results. We selected 21 independent single-nucleotide polymorphisms with genome-wide significance $(p<5.00 \mathrm{E}-08)$ from GWAS on FN BMD as the instrumental variables. The IVW method (beta $=0.010$, standard error $[S E]=0.003, p=0.002$ ) and the weighted median approach (beta $=0.011, \mathrm{SE}=0.004, \mathrm{p}=0.006$ ) yielded evidence of a causal association between FN BMD and OA. However, the MR-Egger analysis showed no causal association between FN BMD and OA (beta $=0.005, \mathrm{SE}=0.017, p=0.753$ ). Since MR-Egger regression suffers from a lack of power and a susceptibility to weak instrument bias, the MR analysis results may support a causal association between FN BMD and OA. Conclusion. The results of MR analysis by IVW and weighted median, but not MR-Egger regression indicate that FN BMD is likely to be causally associated with an increased risk of OA incidence The current findings may provide an opportunity to elucidate the underlying mechanisms of the effects of BMD on the OA incidence. (J Rheum Dis 2019;26:104-110)
\end{abstract}

Key Words. Bone density, Osteoarthritis, Mendelian randomization

\section{INTRODUCTION}

Osteoarthritis (OA) is the most common joint disorder characterized by a progressive degeneration of articular cartilage, joint pain, and immobility [1]. OA is the major cause of pain and disability in elderly people. The prevalence of OA continues to increase worldwide [2] and the health burden of $\mathrm{OA}$ increases with increasing longevity. Therefore, a better understanding of the causal risk factors associated with $\mathrm{OA}$ is needed. Although the etiology of $\mathrm{OA}$ is not fully understood, environmental factors likely play an important role in the development of $\mathrm{OA}$ in genetically susceptible individuals.

Many risk factors including older age, female gender, higher body mass index, and joint injury have been identi- fied to be associated with OA [3,4]. OA is more common in individuals with high bone mineral density (BMD), and observational studies have found that higher BMD is associated with an increased risk of OA $[5,6]$. Although these results suggested a possible common role of bone turnover and repair in manifestations of $\mathrm{OA}$, the mechanism for this action is unclear. In addition, causal association remains unclear, because observational studies are prone to bias, such as reverse causation and residual confounding, thereby precluding a clear understanding of the effects of high BMD on OA [7].

Mendelian randomization (MR) is a technique that uses genetic variants as instrumental variables (IVs) to assess whether an observational association between a risk factor and an outcome is consistent with a causal effect [8].

\footnotetext{
Received : August 17, 2018, Revised : (1st) October 4, 2018, (2nd) November 10, 2018, Accepted : November 22, 2018

Corresponding to : Young Ho Lee (iD http://orcid.org/0000-0003-4213-1909

Department of Rheumatology, Korea University Anam Hospital, Korea University College of Medicine, 73 Inchon-ro, Seongbuk-gu, Seoul 02841, Korea. E-mail : lyhcgh@korea.ac.kr
}

Copyright (c) 2019 by The Korean College of Rheumatology. All rights reserved.

This is an Open Access article, which permits unrestricted non-commerical use, distribution, and reproduction in any medium, provided the original work is properly cited. 
A two-sample MR estimates causal effects where data on the exposure and outcome have been measured in different samples [9]. To the best of our knowledge, MR has not previously been used to explore causal effects of BMD on the incidence of OA. Thus, the aim of this study was to examine whether BMD is causally associated with incidence of OA using a two-sample MR analysis.

\section{MATERIALS AND METHODS}

\section{Data sources and selection of genetic variants}

We searched the MR Base database (http://www.mrbase.org/), which houses a large collection of summary statistics data from hundreds of genome-wide association studies (GWASs). We used publicly available summary statistics dataset of a GWAS on FN BMD of individuals of European ancestry from the general population (up to 49,988 ) as the exposure [10]. Genetic variants associated with femur neck (FN) BMD were used as IVs to improve inference based on p-value threshold of 5.00E-08 (genome-wide significance). We obtained summary statistics (beta coefficients and standard errors) for 21 independent single-nucleotide polymorphisms (SNPs) associated with FN BMD as the IVs from relevant GWAS [10]. We used the publicly available summary statistic datasets of a GWAS for OA from the individuals included in the UK Biobank (up to 46,268 cases and 185,610 controls) (http://www.nealelab.is/uk-biobank/) as the outcome [11]. Both GWAS data on BMD and OA were adjusted for age, sex, and weight $[10,11]$.

Table 1. Instrumental SNPs associated with femur neck BMD and OA GWASs

\begin{tabular}{|c|c|c|c|c|c|c|c|c|c|}
\hline \multirow{2}{*}{$\begin{array}{l}\text { Instrumental } \\
\text { SNP }\end{array}$} & \multirow{2}{*}{ Gene } & \multirow{2}{*}{$\begin{array}{l}\text { Chromosome } \\
\text { loci }\end{array}$} & \multirow{2}{*}{$\begin{array}{l}\text { Effect } \\
\text { allele }\end{array}$} & \multicolumn{3}{|c|}{ Exposure (FN BMD) } & \multicolumn{3}{|c|}{ Outcome (OA) } \\
\hline & & & & Beta & SE & $p$-value & Beta & SE & $\mathrm{p}$-value \\
\hline rs10170839 & CSRNP3 & 2 & $\mathrm{C}$ & -0.059 & 0.008 & $1.20 \mathrm{E}-14$ & -0.001 & 0.001 & 0.032 \\
\hline rs10794639 & AXIN1 & 16 & G & -0.051 & 0.008 & $3.30 \mathrm{E}-11$ & 0.001 & 0.001 & 0.451 \\
\hline rs10946458 & CDKAL1 & 6 & $\mathrm{C}$ & -0.045 & 0.008 & 3.63E-08 & -0.001 & 0.001 & 0.390 \\
\hline rs11024028 & C11ORF58 & 11 & G & 0.056 & 0.010 & $2.18 \mathrm{E}-08$ & 0.001 & 0.001 & 0.303 \\
\hline rs11652763 & HDAC5 & 17 & A & 0.084 & 0.013 & $1.09 \mathrm{E}-10$ & 0.002 & 0.001 & 0.095 \\
\hline rs13194508 & RSPO3 & 6 & $\mathrm{C}$ & -0.052 & 0.009 & $1.30 \mathrm{E}-08$ & 0.001 & 0.001 & 0.193 \\
\hline rs1366594 & MEF2C & 5 & $\mathrm{C}$ & -0.079 & 0.008 & $5.44 \mathrm{E}-25$ & -0.001 & 0.001 & 0.230 \\
\hline rs1485307 & COLEC10 & 8 & $\mathrm{~T}$ & 0.062 & 0.008 & $2.49 \mathrm{E}-15$ & 0.001 & 0.001 & 0.263 \\
\hline rs1785493 & LRP5 & 11 & $\mathrm{~T}$ & -0.045 & 0.008 & 4.06E-08 & 0.000 & 0.001 & 0.545 \\
\hline rs2566752 & GNG12-AS1 & 1 & $\mathrm{C}$ & 0.062 & 0.008 & $3.65 \mathrm{E}-15$ & 0.001 & 0.001 & 0.067 \\
\hline rs2741856 & WHSC1L2P & 17 & $\mathrm{C}$ & 0.088 & 0.014 & $1.34 \mathrm{E}-09$ & 0.001 & 0.001 & 0.432 \\
\hline rs3779381 & WNT16 & 7 & G & 0.058 & 0.009 & $2.87 \mathrm{E}-11$ & 0.001 & 0.001 & 0.183 \\
\hline rs4281029 & STARD3NL & 7 & A & 0.057 & 0.009 & 2.96E-09 & 0.002 & 0.001 & 0.043 \\
\hline rs436448 & CTNNB1 & 3 & $\mathrm{~T}$ & -0.064 & 0.008 & $1.56 \mathrm{E}-16$ & -0.001 & 0.001 & 0.268 \\
\hline rs4448201 & C7orf76 & 7 & G & -0.066 & 0.008 & 4.37E-16 & -0.001 & 0.001 & 0.352 \\
\hline rs4759320 & HOXC4 & 12 & $\mathrm{C}$ & -0.045 & 0.008 & 3.33E-08 & -0.001 & 0.001 & 0.382 \\
\hline rs7108738 & SOX6 & 11 & G & 0.083 & 0.010 & 8.07E-17 & 0.000 & 0.001 & 0.973 \\
\hline rs71390846 & FOXL1 & 16 & $\mathrm{C}$ & -0.059 & 0.010 & $3.16 \mathrm{E}-09$ & 0.000 & 0.001 & 0.958 \\
\hline rs7209460 & SMG6 & 17 & $\mathrm{C}$ & -0.051 & 0.008 & 1.35E-09 & -0.002 & 0.001 & 0.001 \\
\hline rs7524102 & ZBTB40 & 1 & G & 0.084 & 0.010 & $7.36 \mathrm{E}-17$ & 0.000 & 0.001 & 0.604 \\
\hline rs9478217 & CCDC170 & 6 & $A$ & -0.053 & 0.008 & $1.23 \mathrm{E}-11$ & 0.001 & 0.001 & 0.131 \\
\hline
\end{tabular}

SNP: single nucleotide polymorphism, BMD: bone mineral density, OA: osteoarthritis, GWAS: genome-wide association study, FN: femur neck, Beta: beta coefficient, SE: standard error, CSRNP3: cysteine and serine rich nuclear protein 3, AXIN1: axin 1, CDKAL1: CDK5 regulatory subunit associated protein 1 like 1, C11ORF58: chromosome 11 open reading frame 58, HDAC5: histone deacetylase 5, RSPO3: R-spondin 3, MEF2C: myocyte enhancer factor 2C, COLEC10: collectin subfamily member 10, LRP5: LDL receptor related protein 5, GNG12-AS1: GNG12 antisense RNA 1, WHSC1L2P: Wolf-Hirschhorn syndrome candidate 1-like 2, pseudogene, WNT16: Wnt family member 16, STARD3NL: STARD3 N-terminal like, CTNNB1: catenin beta 1, C7orf76: chromosome 7 open reading frame 76, HOXC4: homeobox C4, SOX6: SRY-box 6, FOXL1: forkhead box L1, SMG6: SMG6, nonsense mediated MRNA decay factor, ZBTB40: zinc finger and BTB domain containing 40, CCDC170: coiled-coil domain containing 170 . 


\section{Statistical analysis for Mendelian randomization}

MR analysis requires genetic variants to be related to, but not potential confounders of, an exposure [12]. First, we assessed the independent association of SNPs with FN BMD. Second, we examined the association between each SNP and incidence of OA. Third, we combined these findings to estimate the causal association between FN BMD and incidence of OA using MR analysis. We performed two-sample MR, a method used to estimate the causal effects of an exposure (FN BMD) on outcomes (OA) using summary statistics from different GWASs [13], to assess the causal relationship between FN BMD and incidence of OA, using summary data from GWASs related to FN BMD and OA with 21 SNPs as IVs (Table 1).

The IVW method uses a meta-analysis approach to combine the Wald ratio estimates of the causal effect obtained from different SNPs and provide an estimate of the causal effect of the exposure on the outcome when each of the genetic variant satisfies the assumptions of an instrumental variable [13]. Although the inclusion of multiple variants in an MR analysis results in increased statistical precision, it has the potential to include pleiotropic genetic variants that are not valid IVs [14]. To explore and adjust for pleiotropy, i.e., the association of genetic variants with more than one variable, the weighted median and MR-Egger regression approaches were utilized. MR-Egger regression analysis, which is robust to invalid instruments, tests and accounts for the presence of unbalanced pleiotropy by introducing a parameter for this bias by incorporating summary data estimates of causal effects from multiple individual variants [15]. MR-Egger applies a weighted linear regression of the gene-outcome coefficients on the gene-exposure coefficients [15]. The slope of this regression represents the causal effect estimate, and the intercept can be interpreted as an estimate of the average horizontal pleiotropic effect across the genetic variants [16]. MR-Egger regression was used to assess the potential for violations of MR assumptions and to improve the reliability of causal inference. The weighted median estimator provides a precise estimate of the causal effect, even when up to $50 \%$ of the information contributing to the analysis comes from genetic variants that are invalid IVs [17]. The weighted median estimator has the advantage of retaining greater precision in the estimates compared to the MR-Egger analysis [17]. The analyses were considered statistically significant at $\mathrm{p}$ $<0.05$. All MR analyses were performed on the MR Base platform (app version: 1.2.1 e646be (27 June 2018), R version: 3.5.0) [18].

\section{Heterogeneity and sensitivity test}

We assessed heterogeneities between SNPs using Cochran's Q-statistics [19] and $\mathrm{I}^{2}$ statistic [20,21]. We also performed a "leave-one-out" analysis, where the analysis is re-performed, sequentially excluding one instrument at a time, to test if the result was being driven by any one variant.

\section{RESULTS}

\section{Instrumental variables for Mendelian randomization}

We selected 21 independent SNPs from GWAS on FN BMD as the IVs. All of them are associated with FN BMD with genome-wide significance (Table 1, Figure 1). The F-statistic was used to verify the validity of IVs. A p-value of 5.00E-08 corresponded to $\mathrm{F}>30$ for each single variant [12] and a threshold of $\mathrm{F}<10$ was used to define a "weak IV". Thus, weak instrument bias was negligible. Sixteen of the 21 SNPs were associated with OA, and the association with rs7209460, rs4281029, and rs10170839 was statistically significant and the 3 SNPs also included as IVs (Table 1, Figure 1).

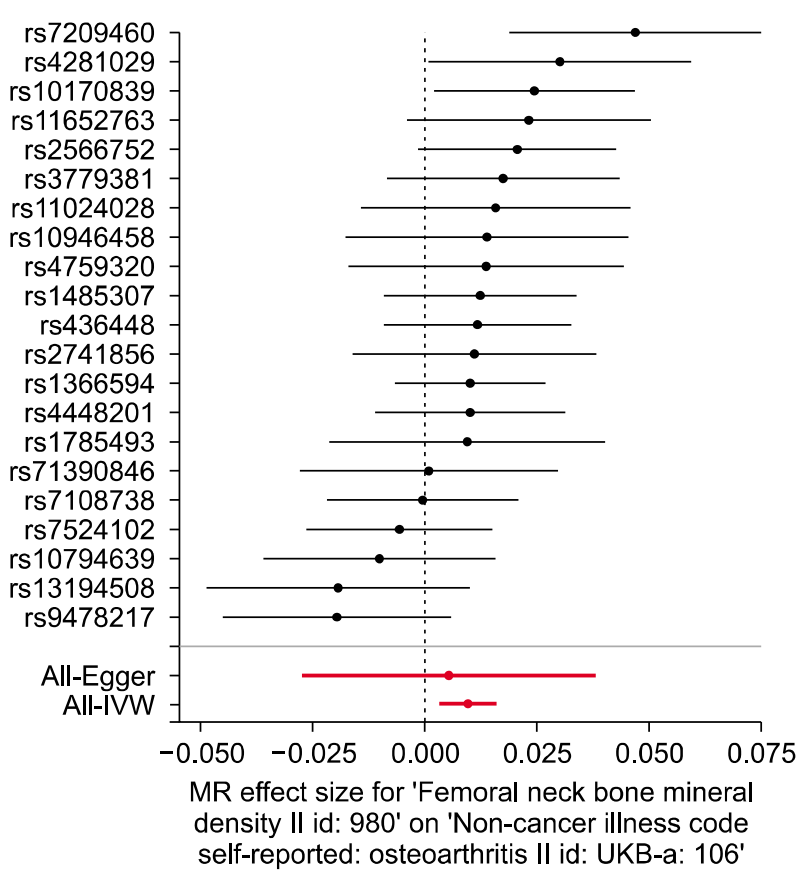

Figure 1. Forest plot of the causal effects of BMD-associated SNPs on OA. BMD: bone mineral density, SNP: single nucleotide polymorphism, OA: osteoarthritis, IVW: inverse-variance weighting, MR: Mendelian randomization. 
Table 2. The MR estimates from each method of assessing the causal effect of femur neck BMD on the incidence of OA

\begin{tabular}{lcccrrrrr}
\hline \hline \multicolumn{1}{c}{ MR method } & $\begin{array}{c}\text { Number of } \\
\text { SNPs }\end{array}$ & Beta & SE & $\begin{array}{c}\text { 95 \% confidence } \\
\text { interval }\end{array}$ & $\begin{array}{c}\text { Association } \\
\text { p-value }\end{array}$ & $\begin{array}{c}\text { Cochran's } \\
\text { Q statistic }\end{array}$ & $\begin{array}{c}\text { I }^{2} \\
\text { Heterogeneity } \\
\text { p-value }\end{array}$ \\
\hline Inverse variance weighted & 21 & 0.010 & 0.003 & $0.004 \sim 0.016$ & 0.002 & 27.39 & 0.262 & 0.125 \\
MR Egger & 21 & 0.005 & 0.017 & $-0.028 \sim 0.038$ & 0.753 & 27.28 & 0.304 & 0.098 \\
Weighted median & 21 & 0.011 & 0.004 & $0.003 \sim 0.019$ & 0.006 & $27.10^{*}$ & $0.270^{*}$ & $0.133^{*}$ \\
\hline
\end{tabular}

MR: Mendelian randomization, BMD: bone mineral density, OA: osteoarthritis, SNP: single nucleotide polymorphism, Beta: beta coefficient, SE: standard error. *Maximum likelihood method, $I^{2}=(Q-d f) / Q[21]$.

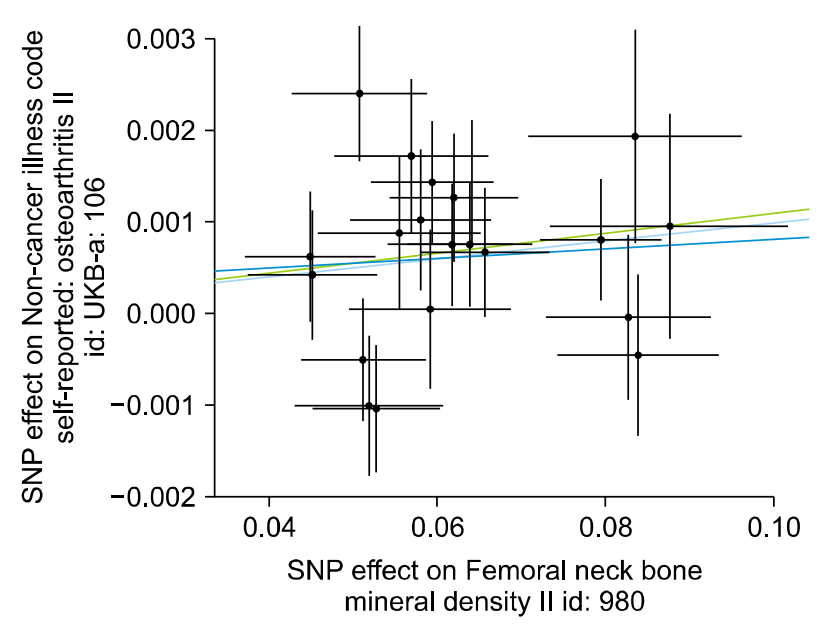

Figure 2. Scatter plots of genetic associations with BMD against the genetic associations with OA. The slopes of each line represent the causal association for each method. The blue line represents the inverse-variance weighting estimate, the green line represents the weighted median estimate, and the dark blue line represents the Mendelian randomizationEgger estimate. BMD: bone mineral density, OA: osteoarthritis, SNP: single nucleotide polymorphism.

\section{Mendelian randomization results}

The IVW method showed evidence to support a causal association between FN BMD and OA (beta=0.010, standard error $[S E]=0.003, p=0.002$, OR per genetically predicted 1 standard deviation in FN BMD=1.011) (Table 2 , Figures 1 and 2). The intercept represents the average pleiotropic effect across the genetic variants (the average direct effect of a variant with the outcome). If the intercept differs from zero (the MR-Egger test), then there is an evidence of directional pleiotropy. MR-Egger regression analysis revealed that directional pleiotropy was unlikely to bias the result (intercept $=0.0003 ; \mathrm{p}=0.784$ ). The MR-Egger analysis showed no causal association between FN BMD and OA (beta $=0.005, \mathrm{SE}=0.017$, $\mathrm{p}=0.753$ ) (Table 2, Figures 1 and 2). However, the weighted median approach yielded evidence of a causal association between FN BMD and OA (beta=0.011,

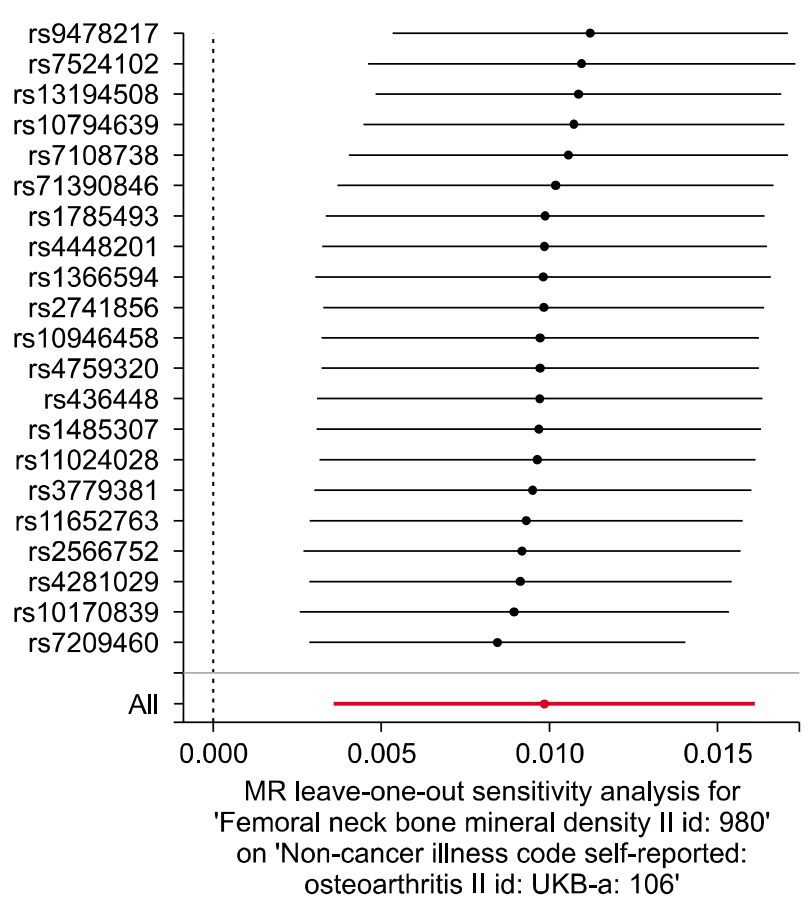

Figure 3. "Leave-one-out" analysis to investigate the possibility that the causal association was driven by a unique SNP. SNP: single nucleotide polymorphism, MR: Mendelian randomization.

$\mathrm{SE}=0.004, \mathrm{p}=0.006$ ) (Table 2, Figure 2). Thus, IVW and weighted median method suggested a causal effect of FN BMD on OA risk, whereas the MR-Egger method suggests a null causal effect. Considering that the weighted median estimator has the advantage of retaining greater precision compared to the MR-Egger analysis [17], it may be suggested the MR analysis results support a causal association between FN BMD and OA. The common risk factor for both high BMD and OA is obesity. Thus obesity is a major confounding factor for this analysis. Therefore, we checked whether significantly associated SNPs with OA including rs7209460, rs4281029, and rs10170839 were associated with obesity. We did a literature search on the association between leading SNPs and obesity through searching PUBMED and GWAS results database 
(https://www.ebi.ac.uk/gwas/). There was no evidence of their associations with obesity, indicating obesity may not act as confounding factor in this MR analysis.

\section{Heterogeneity and sensitivity test}

Cochran's Q test indicated no evidence of heterogeneity between IV estimates based on the individual variants (Table 2). Heterogeneity is the variability in the causal estimates obtained for each SNP (i.e., how consistent is the causal estimate across all SNPs). Low heterogeneity suggested increased reliability of MR estimates. $\mathrm{I}^{2}$ values showed low heterogeneity, indicating increased reliability of MR estimates (Table 2). Results from the "leave-one-out" analysis demonstrated that no single SNP was driving the IVW point estimate (Figure 3).

\section{DISCUSSION}

BMD has been considered to be a risk factor for OA $[5,6,22]$. However, it remains unclear whether BMD has a causal relationship with $\mathrm{OA}$, because previously reported associations between BMD and osteoarthritis may be the result of bias or confounding factors inherent to observational studies, such as body weight, physical activity, reverse causation, a small number of studies of small sizes, and selection bias [7]. MR minimizes the possibility of bias inherent to observational studies [23]. Thus, we carried out three different estimating methods (inverse variance weighting method, weighted median method, and MR-Egger regression) for the MR analyses. Our study indicated that the associations between FN BMD and OA may be causal. Although the MR estimates using IVW, MR Egger, and weighted median analysis were not consistent. IVW and weighted median analysis supported a causal association between FN BMD and OA, whereas MR Egger analysis did not. As all the variants used in MR may not be the valid instruments, pleiotropy robust tools for sensitivity analysis including MR-Egger regression and the weighted median approach have been developed. Both MR-Egger and the weighted median should be considered as a tool for sensitivity analysis rather than as a primary analysis tool. However, unfortunately, MR-Egger regression suffers from a lack of power, a susceptibility to weak instrument bias, and low precision. Considering these limitations of MR-Egger regression [17], a weighted median may be a better way to adjust pleiotropy than MR-Egger regression. Thus, our study corroborated the association found in previous ob- servational studies [5,6,22].

MR studies are susceptible to bias from pleiotropy (association of genetic variants with more than one variable) [24]. Although the inclusion of multiple variants in MR analysis typically leads to increased statistical precision, it also results in the potential inclusion of pleiotropic genetic variants that are not valid IVs [25]. Therefore, the approaches for the sensitivity analysis need to be applied to verify the validity of conclusions drawn from the MR study. To eliminate pleiotropy, we employed a weighted median estimator, which provides valid estimates even if $50 \%$ of the SNPs are not valid instruments [17], and we used MR-Egger regression approach to provide a test for unbalanced pleiotropy and a causal estimate of the influence of exposure on outcome in its presence [15]. Our results using all three approaches were not consistent, but the MR-Egger approach showed no evidence for unbalanced pleiotropy as indicated by intercept p-value. The MR-Egger method led to loss of precision and power, but our weighted median estimator results were also similar to the IVW estimator, thereby providing additional confidence for these associations. Our data was in agreement with the previous observational studies, which have shown an association between BMD and OA. Increased OA risk may be a direct consequence of high BMD. Probability of OA incidence may be enhanced as a direct consequence of high BMD due to mechanical pressure applied to cartilage from the underlying subchondral cortical plate [26].

MR rests on three assumptions of the InSIDE (instrument strength independent of direct effect). The first assumption was evaluated directly by examining the strength of association of the IV SNPs with exposure (FN $\mathrm{BMD})$. We proved the second assumption by checking that significantly associated SNPs with OA were not associated with obesity. Third assumption was evaluated by MR-egger regression test, which indicated that directional pleiotropy was unlikely to bias the result. Our MR analysis may hold the InSIDE assumptions and the causal estimate may be reliable.

The present study has several limitations. First, our analysis included a relatively small number of SNPs as IVs, which might not have been statistically significant enough to detect an association; the statistical precision can be increased and a more precise causal estimate can be obtained by combining multiple genetic variants together. In addition, OA was included in this MR analysis regardless of OA site. Subgroup analysis by OA site 
showed a trend of causal relationship between BMD and OA due to decreased sample size in each subgroup. Thus, we combined all samples into one OA group to improve statistical power, because observational studies showed associations between high BMD and OA risk of knee and hip $[5,6,22]$. Finally, individuals with OA were classified by non-cancer illness code self-reported, which might cause potential bias, such as selection bias and information bias, compared to radiographic OA criteria.

Unlike observational studies on associations between high BMD and higher risk of OA [5,6,22], our study was not susceptible to bias from unmeasured confounders or reverse causation by adopting MR approaches, and demonstrated that FN BMD may play a causal role in OA etiology. MR analysis presented in this study was reliable. Although BMD has been studied as a potential risk factor for OA, an MR study has been never performed. This is the first study on the causal relationship between BMD and OA.

\section{CONCLUSION}

In conclusion, the results of MR analysis by IVW and weighted median, but not MR-Egger regression indicate that FN BMD is likely to be causally associated with an increased risk of $\mathrm{OA}$ incidence. The current findings may provide an opportunity to elucidate the underlying mechanisms of the effects of BMD on the OA incidence.

\section{CONFLICT OF INTEREST}

No potential conflict of interest relevant to this article was reported.

\section{REFERENCES}

1. Dieppe PA, Lohmander LS. Pathogenesis and management of pain in osteoarthritis. Lancet 2005;365:965-73.

2. Litwic A, Edwards MH, Dennison EM, Cooper C. Epidemiology and burden of osteoarthritis. Br Med Bull 2013; 105:185-99.

3. Silverwood V, Blagojevic-Bucknall M, Jinks C, Jordan JL, Protheroe J, Jordan KP. Current evidence on risk factors for knee osteoarthritis in older adults: a systematic review and meta-analysis. Osteoarthritis Cartilage 2015;23:507-15.

4. Park EH, Kim JS, Lee JS, Lee YJ, Song YW, Lee EY. Compound $\mathrm{K}$ inhibits interleukin- $1 \beta$-induced expression of inflammatory mediators and matrix metalloproteinases by inhibiting mitogen-activated protein kinase activation in chondrocytes. J Rheum Dis 2018;25:188-96.
5. Hart DJ, Cronin C, Daniels M, Worthy T, Doyle DV, Spector TD. The relationship of bone density and fracture to incident and progressive radiographic osteoarthritis of the knee: the Chingford study. Arthritis Rheum 2002;46:92-9.

6. Nevitt MC, Zhang Y, Javaid MK, Neogi T, Curtis JR, Niu J, et al. High systemic bone mineral density increases the risk of incident knee OA and joint space narrowing, but not radiographic progression of existing knee OA: the MOST study. Ann Rheum Dis 2010;69:163-8.

7. Ranstam J. Bias in observational studies. Acta Radiol 2008; 49:644-5.

8. Burgess S, Daniel RM, Butterworth AS, Thompson SG; EPIC-InterAct Consortium. Network Mendelian randomization: using genetic variants as instrumental variables to investigate mediation in causal pathways. Int J Epidemiol 2014;44:484-95.

9. Lawlor DA. Commentary: two-sample Mendelian randomization: opportunities and challenges. Int J Epidemiol 2016; 45:908-15.

10. Zheng HF, Forgetta V, Hsu YH, Estrada K, Rosello-Diez A, Leo PJ, et al. Whole-genome sequencing identifies EN1 as a determinant of bone density and fracture. Nature 2015; 526:112-7.

11. Zengini E, Hatzikotoulas K, Tachmazidou I, Steinberg J, Hartwig FP, Southam L, et al. Genome-wide analyses using UK Biobank data provide insights into the genetic architecture of osteoarthritis. Nat Genet 2018;50:549-58.

12. Burgess S, Butterworth A, Thompson SG. Mendelian randomization analysis with multiple genetic variants using summarized data. Genet epidemiol 2013;37:658-65.

13. Pierce BL, Burgess S. Efficient design for Mendelian randomization studies: subsample and 2-sample instrumental variable estimators. Am J Epidemiol 2013;178:1177-84.

14. Hartwig FP, Davies NM, Hemani G, Davey Smith G. Two-sample Mendelian randomization: avoiding the downsides of a powerful, widely applicable but potentially fallible technique. Int J Epidemiol 2016;45:1717-26.

15. Bowden J, Davey Smith G, Burgess S. Mendelian randomization with invalid instruments: effect estimation and bias detection through Egger regression. Int $\mathrm{J}$ Epidemiol 2015;44:512-25.

16. Burgess S, Thompson SG. Interpreting findings from Mendelian randomization using the MR-Egger method. Eur J Epidemiol 2017;32:377-89.

17. Bowden J, Davey Smith G, Haycock PC, Burgess S. Consistent estimation in Mendelian randomization with some invalid instruments using a weighted median estimator. Genet Epidemiol 2016;40:304-14.

18. Hemani G, Zheng J, Elsworth B, Wade KH, Haberland V, Baird D, et al. The MR-base platform supports systematic causal inference across the human phenome. Elife 2018:7. pii: e34408.

19. Egger M, Smith GD, Phillips AN. Meta-analysis: principles and procedures. BMJ 1997;315:1533-7.

20. Bowden J, Del Greco M F, Minelli C, Davey Smith G, Sheehan NA, Thompson JR. Assessing the suitability of summary data for two-sample Mendelian randomization analyses using MR-Egger regression: the role of the $\mathrm{I}^{2}$ statistic. Int J Epidemiol 2016;45:1961-74.

21. Higgins JP, Thompson SG. Quantifying heterogeneity in a meta-analysis. Stat Med 2002;21:1539-58. 
22. Nevitt MC, Lane NE, Scott JC, Hochberg MC, Pressman AR, Genant HK, et al. Radiographic osteoarthritis of the hip and bone mineral density. The study of osteoporotic fractures research group. Arthritis Rheum 1995;38:907-16.

23. Smith GD, Ebrahim S. Mendelian randomization: genetic variants as instruments for strengthening causal inference in observational studies. In: Weinstein M, Vaupel JW, Wachter KW, National Research Council (U.S.). Committee on Advances in Collecting and Utilizing Biological Indicators and Genetic Information in Social Science Surveys., National Research Council (U.S.). Committee on Population, 2006-2007, eds. Biosocial surveys. Washington, D.C., National Academies Press, 2008. 24. Thompson JR, Minelli C, Bowden J, Del Greco FM, Gill D, Jones EM, et al. Mendelian randomization incorporating uncertainty about pleiotropy. Stat Med 2017;36:4627-45.

25. Smith GD, Ebrahim S. Mendelian randomization: prospects, potentials, and limitations. Int J Epidemiol 2004;33: $30-42$.

26. Lo GH, Niu J, McLennan CE, Kiel DP, McLean RR, Guermazi A, et al. Meniscal damage associated with increased local subchondral bone mineral density: a Framingham study. Osteoarthritis Cartilage 2008;16:261-7. 